\title{
Coproantigen Versus Classical Microscopy as a Diagnostic Tool for Entamoeba histolytica Infection in the Egyptian Patients
}

\author{
Ahmed M.S. Bayoumy' ${ }^{1}$ Mohsen T.I. Elkeiy ${ }^{2}$, Tarek K.I .Zaalok ${ }^{1}$, Hesham M. Gad ${ }^{3}$, \\ Wael A.M. Abd Elhamid' \\ ${ }^{1}$ Department of Parasitology, ${ }^{2}$ Department of Pediatrics, Faculty of Medicine, ${ }^{3}$ Department of Biochemistry, \\ Faculty of Pharmacy, Al-Azhar University, Cairo, Egypt \\ Corresponding author: Ahmed M.S. Bayoumy, e-mail: drahmedbayoumy@azhar.edu.eg
}

\begin{abstract}
Background: Entamoeba histolytica, an amoebic protozoan, is considered as one of the most common causes of nonviral (Parasitic) diarrheal illness in humans. Laboratory diagnosis consists primarily of direct microscopic examination of stool specimen for both trophozoites and cysts. However, because of the intermittent fecal excretion of the parasite, the case may be mis-diagnosed and the patient may continue excreting the parasite and infecting others. That is why other methods of diagnosis should be looked for that can help overcome the defects and drawbacks of microscopy when used alone for diagnosis. Aim of the work: the current study aimed to evaluate the efficacy of coproantigen detection by ELISA test in comparison with direct microscopy in diagnosis of $E$. histolytica/dispar in stool specimens from patients with diarrhea and other gastrointestinal symptoms.

Material and Methods: stool samples were collected form 250 children included in the present study (150 symptomatic and 100 asymptomatic groups) between the ages of 1 and 10 years and subjected to direct microscopic examination and ELISA test for coproantigen detection.

Results: out of 250 stool samples, 64 specimens (25.6\%) were positive for E. histolytica/dispar by direct microscopy, while 79 specimens $(31.6 \%)$ were positive by ELISA test. The sensitivity and specificity of ELISA test compared to direct microscopy were found to be $96.9 \%$ and $90.9 \%$, respectively. Conclusion: ELISA test for coproantigen detection in stool samples is a rapid and effective method with high sensitivity and specificity for diagnosis of amoebiasis in stool specimens even when the parasitic count is low, thus reducing the chances of missing positive cases even in the asymptomatic cases.
\end{abstract}

Keywords: Entamoeba histolytica, microscopic stool examination, coproantigen.

\section{INTRODUCTION}

Amoebiasis is a human infection, which is caused by Entamoeba histolytica, a protozoan of cosmopolitan distribution, with or without clinical manifestations ${ }^{(\mathbf{1})}$. It affects more than 50 million people worldwide and is considered as the most common parasitic infection specifically in the tropics and subtropics ${ }^{(2)}$.

It is an important cause of morbidity and mortality worldwide (3) mainly in developing countries, where sanitation infrastructure and health services are often insufficient ${ }^{(4)}$. Although the distribution of the parasite is worldwide, the preponderance of morbidity and mortality is experienced in the Central and South America, Africa, and India ${ }^{(5)}$.

Children and young adults are the most affected group, specifically in regions with limited resources and in areas with low hygienic measures ${ }^{\left({ }^{6}\right.}$. Humans are the main host of E. histolytica and there are no other known animal reservoirs of this parasite ${ }^{(7)}$ and most of the infected persons are carriers ${ }^{(8)}$. The infection is responsible for a considerable number of cases of prolonged diarrhea in travelers ${ }^{(9)}$. In addition, infection with $E$. histolytica may lead to the development of life- threatening abscess in liver, brain or lungs ${ }^{(\mathbf{1 0})}$. Waterassociated outbreaks of E. histolytica disease had been reported ${ }^{(\mathbf{1 1})}$ and sexual transmission was also recorded (12). Clinical features of amoebiasis range from asymptomatic colonization to amoebic colitis (Dysentery or diarrhea) and the invasive extraintestinal infection, which manifests most commonly in the form of liver abscess ${ }^{(\mathbf{1 2})}$.

The traditional method of diagnosing intestinal infection by microscopic examination of fresh stool samples was only 50-60\% sensitive and can give false positive results. This is because E. histolytica is microscopically indistinguishable from the morphologically identical nonpathogenic species, Entamoeba dispar and Entamoeba moshkovskii (13).

A correct diagnosis of infection is, however, necessary to avoid undue treatment for amoebiasis of patients infected with the nonpathogenic species, so WHO stressed on the urgent need to develop improved methods for specific diagnosis of $E$. histolytica infection in the developing countries ${ }^{(\mathbf{1})}$. 
Compared to the sensitivities of enzyme-linked immunosorbent assay (ELISA) antigen in stool and the traditional PCR, real-time PCR has proven to be the most sensitive test for the detection of E. histolytica in stool (14). Real-time PCR is not easy for routine diagnosis because expensive equipments and specialized personnel are required to complete the analysis of the results. For this reason, using ELISA to detect antigen and antibody becomes the standard method to diagnose E. histolytica infection ${ }^{(14)}$.

\section{MATERIALS and METHODS}

A group of 150 children were included in the current study, from those attending the outpatient clinics of pediatrics at Al-Hussein and Said Galal University Hospitals, Faculty of Medicine, Al-Azhar University, Cairo, Egypt, between the ages of 1 and 10 years, complaining of gastrointestinal symptoms as abdominal pain, vomiting, diarrhea, indigestion, distension, dehydration and weight loss (Symptomatic group). Also, another group of 100 apparently healthy children was selected as an asymptomatic group. This study was conducted over a period of 13 months, from December 2016 to December 2017. Stool samples were collected from every child and a written informed consent was taken from the child's parents before the collection of samples.

Stool samples were collected in a $25 \mathrm{ml}$ clean, dry widemouthed plastic container. Gross examination was performed for color, consistency, mucus, blood and adult parasites. Each sample was divided into 2 parts: the first part was used to prepare slides for direct wet smear examination and formalin-ethyl acetate sedimentation concentration method according to Garcia et al. ${ }^{(15)}$ while, the second part was immediately stored at $-20^{\circ} \mathrm{C}$ for coproantigen detection.

\section{Coproantigen detection by ELISA:}

It was performed by using Wampole ${ }^{\mathrm{TM}} E$. histolytica II A $2^{\text {nd }}$ generation Monoclonal ELISA kit for detecting E. histolytica adhesion in fecal specimens (TechLab, Blacksburg, Virginia, USA). The test was carried out according to the manufacturer's instructions. Statistical analysis was performed by using direct microscopy as the gold diagnostic standard. The Wampole ${ }^{\mathrm{TM}} E$. histolytica II A 2nd generation Monoclonal ELISA kit was evaluated for sensitivity, specificity, and positive predictive value using SPSS (Version 18).

\section{RESULTS}

Out of the 150 symptomatic children, 52 of them were positive for amoebiasis by direct microscopy, while 63 children were positive by ELISA test. Among the 100 children representing the asymptomatic group, 12 children were positive by direct microscopy, while 16 children were positive by ELISA test (Tables 1 and 2 and Figures 1 and 2).

Table 1: comparison between asymptomatic and symptomatic groups regarding direct microscopical examination

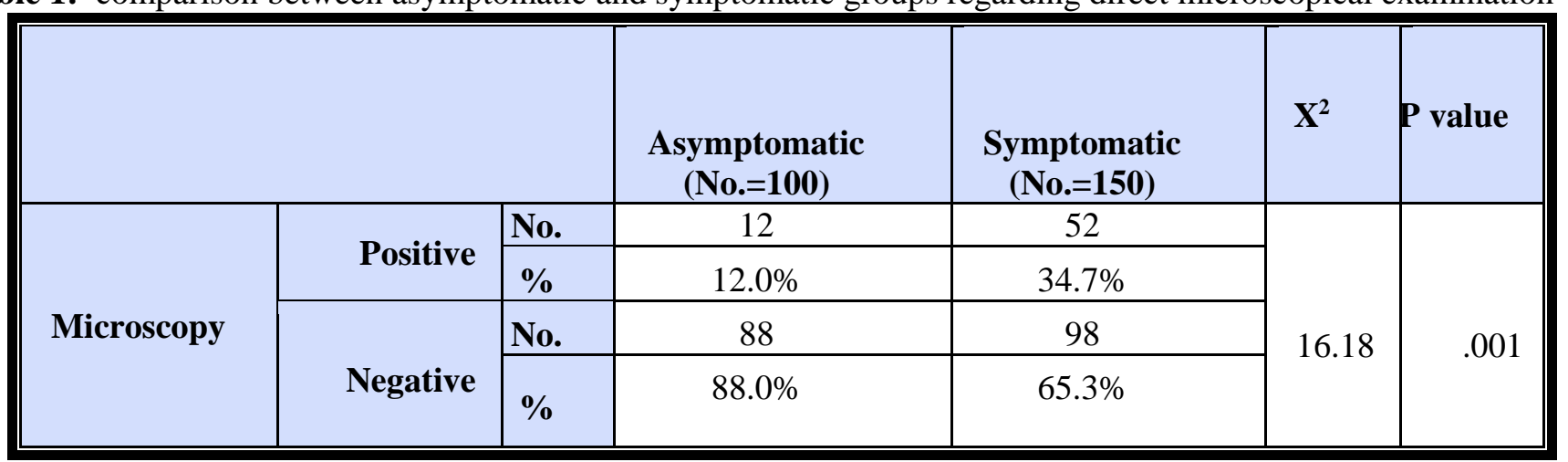

$\%$ of positive specimens was significantly lower among asymptomatic than symptomatic groups (12.0\% and 34.7\%, resp.). $\mathrm{P}=.000$. 


\section{Direct Microscopical Examination}

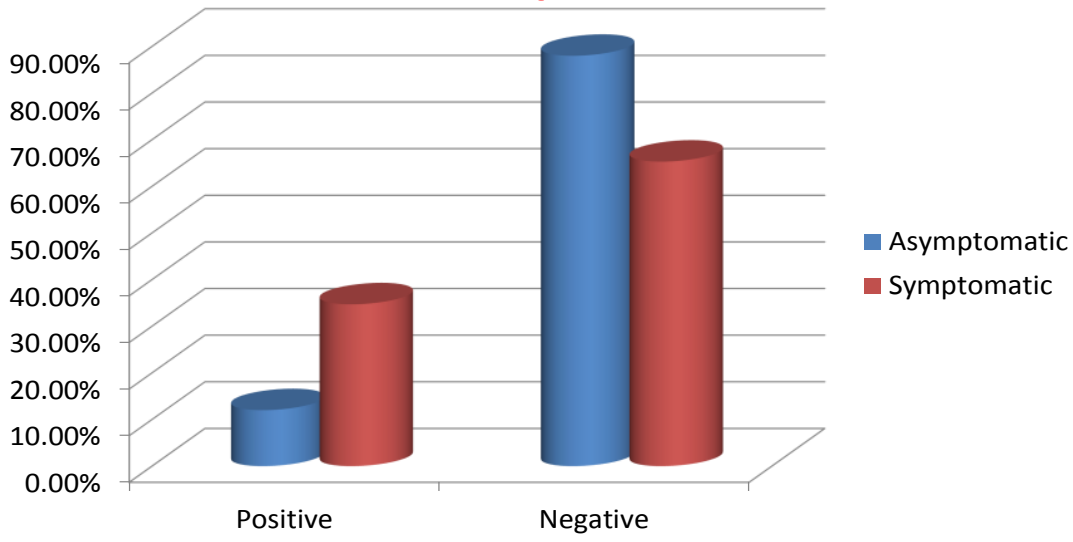

Figure 1: comparison between asymptomatic and symptomatic groups regarding direct microscopical examination.

Table 2: comparison between asymptomatic and symptomatic groups regarding ELISA (Coproantigen)

\begin{tabular}{|c|l|c|c|c|l|l||}
\hline \multicolumn{2}{|c|}{} & $\begin{array}{c}\text { Asymptomatic } \\
(\text { No.=100) }\end{array}$ & $\begin{array}{c}\text { Symptomatic } \\
(\text { No.=150) }\end{array}$ & \multirow{2}{*}{$X^{2}$} & P value \\
\hline \multirow{3}{*}{ ELISA } & \multirow{3}{*}{ Positive } & No. & 16 & 63 & 15.77 & .001 \\
\cline { 3 - 6 } & \multirow{2}{*}{ Negative } & No. & $16.0 \%$ & $42.0 \%$ & & \\
\cline { 2 - 6 } & & $\%$ & 84 & 87 & \\
\hline
\end{tabular}

$\%$ of positive specimens was significantly lower among asymptomatic than symptomatic groups (16.0\% and $42.0 \%$, resp.). $\mathrm{P}=.000$.

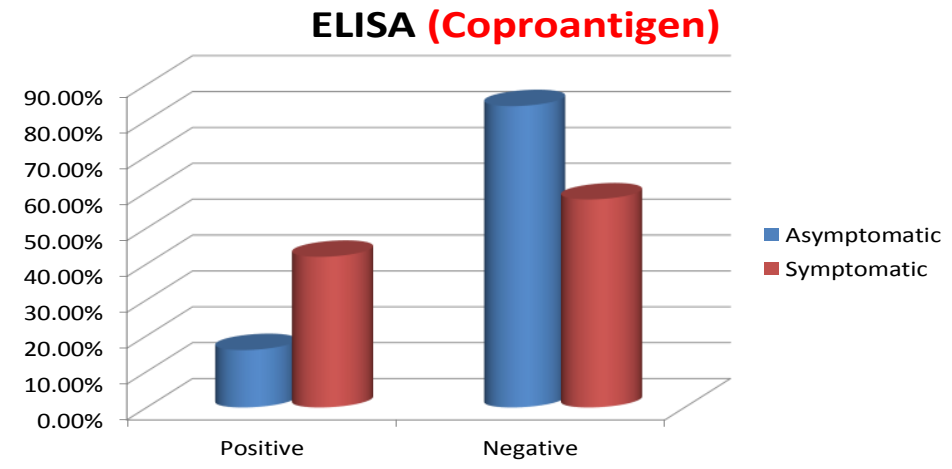

Figure 2: comparison between asymptomatic and symptomatic groups regarding ELISA (Coproantigen) 


\section{DISCUSSION}

Amoebiasis is a human infection that caused by Entamoeba histolytica, a protozoan of cosmopolitan distribution, with or without clinical manifestations, while the infection by $E$. dispar is approximately ten times more ${ }^{(\mathbf{1})}$. Due to the morphological similarity of both species, diagnosis based on light microscopy can yield either under- or overestimation of infection rates, leading to unnecessary treatment ${ }^{\left({ }^{(16)}\right.}$. Due to the invasive behavior of $E$. histolytica and the noninvasive nature of $E$. dispar, coupled with the inability of microscopy to distinguish between both species. World Health Organization (WHO) recommended that diagnoses attained by microscopy must be recorded as "E.histolytica / E.dispar". Also, the WHO recommended procedures that are capable of ensuring differentiation between the two species so that treatment is restricted only for cases of $E$. histolytica infection ${ }^{(\mathbf{1})}$.

Immunological, biochemical and molecular biology methods are currently capable of differentiating between Entamoeba species. Among these methods, tests for antigen detection in stool samples were advantageous in terms of speed, accuracy, and reliability (16). So the current study aimed to evaluate the efficacy of coproantigen detection by ELISA test in comparison with direct microscopy in the diagnosis of amoebiasis.

In the present work, the prevalence of Entamoeba histolytica/dispar was $25.6 \%$ by direct microscopy (64 out of 250 cases) and as high as $31.6 \%$ by ELISA ( 79 out of 250 cases). These results are coincided with that obtained by Ibrahim et al. ${ }^{(17)}$ but the results were lower than that obtained by ElHamshary et al. ${ }^{(18)}$ who found that $54.8 \%$ of their cases were positive by microscopy, while $52.7 \%$ were detected by Entamoeba coproantigens. Also, the results were higher than that obtained by El-Shazly $\boldsymbol{e} t$ al. (19) who found the prevalence rate was $19 \%$ in Mansoura, Egypt.

The difference in results may be due to the different study area, sample size, age group, and environmental, socioeconomic, demographic, and host hygiene related behavioral factors as well as methods of stool examination.

In the current study, the sensitivity and specificity of ELISA test in comparison with direct microscopy were found to be $96.9 \%$ and $90.9 \%$, respectively. This is coincided with results of ElHamshary et al. (18) where the sensitivity and specificity reached $88.24 \%$ and $90.48 \%$, respectively. In another German study, the sensitivity and specificity of ProSpecT ELISA were $73.5 \%$ and $97.7 \%$ in stool specimens, respectively, compared to microscopy for E. histolytica/E.dispar in travelers returning from vacations abroad ${ }^{(20)}$. Haque et al. ${ }^{(21)}$ reported that the overall correlation between results of the Tech Lab antigen detection test and PCR was 94\%. In another study, ELISA test was compared with microscopy for identification of E. histolytica and showed ELISA to be $96 \%$ sensitive and 93\% specific as compared to stool microscopy ${ }^{(22)}$, while Haque et al. ${ }^{(23)}$ showed ELISA to be $97 \%$ specific and $100 \%$ sensitive.

This means that ELISA is a very good sensitive diagnostic test for detection of the disease. However, lower specificity may be due to some cross-reactions with other intestinal parasites or past infection with amoebiasis. So, if ELISA result is negative, it can be fairly said that the patient does not have amoebiasis.

\section{CONCLUSION}

Although direct microscopy is considered as a gold standard test for diagnosis of amoebiasis, its sensitivity ranged from $5 \%$ to $60 \%$ and its specificity ranged from $10 \%$ to $50 \%$. Also, it may give false negative results, especially in chronic infection due to intermittent shedding of cysts, but $2^{\text {nd }}$ generation of Monoclonal ELISA kit is considered as a rapid and effective method with high sensitivity and specificity in detecting Entamoeba histolytica antigens in stool specimens even when the parasitic count is low, thus reducing the chances of missing positive cases even in the asymptomatic (Carrier) cases. It is easier to perform and is useful for rapid investigation of large number of stool specimens.

\section{REFERENCES}

1. WHO (1997): A consultation with experts on amoebiasis: Mexico City, Mexico 28-29. Epidemiol Bull., 18(1): 1314.

2. Nuran D, Gonal A, Mehmet S, Babur C, Kanik A, and Emekdas G (2004): Detection of Entamoeba histolytica/Entamoeba dispar in stool specimens by using enzyme linked immunosorbent assay. Mem. Inst. Oswaldo Cruz., 99: 769-772.

3. Choudhuri G and Rangan M (2012): Amebic infection in humans. Indian J. Gastroenterol., 31 (4): 153-162

4. Ximenez C, Moran P, Rojas L, Valadez A and Gomez A (2009): Reassessment of the epidemiology of amebiasis: state of the art. Infect. Genet. Evol., 9: 1023-1032. 
5. Parija SC, Mandal J, and Ponnambath DK (2014): Laboratory methods of identification of Entamoeba histolytica and its differentiation from look-alike Entamoeba spp. Trop. Parasitol., 4: 90-95.

6. Guerrant RL, Oria R, Bushen OY, Patrick PD, Houpt E and Lima AA (2005): Global impact of diarrheal diseases that are sampled by travelers: the rest of the hippopotamus. Clin. Infect Dis., 41(8):524-530

7. Tengku SA and Norhayati M (2011): Public health and clinical importance of amoebiasis in Malaysia: a review. Trop. Biomed., 28 (2): 194-222.

8. Nair GV and Variyam EP (2014): Noninvasive intestinal amebiasis: Entamoeba histolytica colonization without invasion. Curr. Opin. Infect. Dis., 27(5): 465-469.

9. Slack A (2012): Parasitic causes of prolonged diarrhea in travellers - diagnosis and management. Aust. Fam. Physician, 41(10): 782-786.

10. Petri WA and Haque R (2013): Entamoeba histolytica brain abscess. Clin. Neurol., 114: 147-152.

11. Karanis P, Kourenti C and Smith $H$ (2007):

Waterborne transmission of protozoan parasites: a worldwide review of outbreaks and lessons learnt. J. Water Health, 5 (1): 1-38.

12. Showler AJ and Boggild AK (2013): Entamoeba histolytica. CMAJ., 185(12): 1064-1073.

13. Fotedar R, Stark D, Beebe N, Marriott D, Ellis $\mathbf{J}$ and Harkness J (2007): Laboratory diagnostic techniques for Entamoeba species. Clin. Microbiol. Rev., 20: 512-532.

14. Stark D, van Hal S, Fotedar R et al. (2008): Comparison of stool antigen detection kits to PCR for diagnosis of amebiasis. J. Clin. Microbiol., 46: 1678-1681.

15. Garcia LS, Arrowood M, Kokoskin E, Paltridge GP, Pillai DR, Procop GW, Ryan N, Shimizu RY and Visvesvara G (2017): Laboratory diagnosis of parasites from the gastrointestinal tract. Clin. Microbiol. Rev., 31(1):15-22.

16. Delialioglu N, Aslan G, Sozen M, Babur C, Kanik A and Emekdas G (2004): Detection of Entamoeba
histolytica/Entamoeba dispar in stool specimens by using Enzyme linked immunosorbent assay. Mem. Inst. Oswaldo. Cruz., 99: 769-772.

17. Ibrahim SS, El-Matarawy OM, Ghieth MA, Abu Sarea EY, and El-Badry AA (2015): Copro prevalence and estimated risk of Entamoeba histolytica in diarrheic patients at Beni-Suef, Egypt. World J. Microbiol. Biotechnol., 31: 385-390

18. El-Hamshary EM, El-Shewy KA, Hegazy MM and Zakaria H. (2004): Diagnostic potentials of coproantigen detection based ELISA, compared to microscopy in intestinal amoebiasis. J. Egypt. Soc. Parasitol., 34(2): 601610.

19. El-Shazly AM, Awad SE, Sultan DM, Sadek GS, Khalil HH and Morsy TA (2006): Intestinal parasites in Dakahlia Governorate, with different techniques in diagnosing protozoa. J. Egypt Soc. Parasitol., 36(3): 10231034

20. Jelinek T, Peyerl GT, Löscher and Nothdurft HD (1996): Evaluation of an antigen-capture enzyme immunoassay for detection of Entamoeba histolytica in stool samples. Eur. J. Clin. Microbiol. Infect. Dis., 15: 752755 .

21. Haque R, Ali IK, Akther S and Petri WA (1998): Comparison of PCR, isoenzyme analysis and antigen detection for diagnosis of Entamoeba histolytica infection. J. Clin. Microbiol., 36: 449-452.

22. Haque R, Laurie M, Neville, Sheila $W$ and Petri WA (1994): Short report: detection of Entamoeba histolytica and E. dispar directly in dtool. Am. J. Trop. Med. Hyg., 50(5): 595-596.

23. Haque R, Kress K, Wood S, Jackson TF, Lyerly D, Wilkins T and Petri WA (1993): Diagnosis of pathogenic Entamoeba histolytica infection using a stool ELISA based on monoclonal antibodies to the galactose-specific adhesion. J. Infect. Dis., 168(2): 513-515. 